\title{
Libelium-based IoT Monitoring Solution for Precision Agriculture
}

\author{
Cristina Balaceanu ${ }^{1}$, George Suciu ${ }^{1}$, Ioana Marcu ${ }^{1}$ \\ ${ }^{1}$ Research Department \\ Beia Consult International \\ Peroni Street, no 16, Bucharest, Romania \\ cristina.balaceanu@beia.ro,george@beia.ro \\ ${ }^{2}$ University Politechnica of Bucharest \\ Splaiul Independentei \\ No. 313, Bucharest, Romania \\ ioana.marcu@upb.ro
}

\begin{abstract}
The Internet of Things (IoT) is quite used in Smart Agriculture because of the use of IoT sensors that can provide information related to the agricultural fields on which the crops are growing. This paper aims to illustrate the results achieved using evolutionary technologies, i.e., IoT in precision agriculture to monitor environmental factors and to improve the efficiency of efficient crops. Based on significant characteristics of cloud computing and key techniques of IoT technology, Libelium-based IoT platform can help building up an enormous database comprising numerous agricultural production parameters. The main feature of this paper consists in outlining the monitoring of temperature, humidity of soil, relative humidity, atmospheric pressure and solar radiation in the agricultural field through sensors using the Libelium station. By means of data transmission protocol, vital information will reach farmers using Wi-Fi technology, information based on which they can decide if their crops need irrigation or not.
\end{abstract}

Keywords: Precision Agriculture, IoT, Libelium Platform, Sensors

Received: 18 October 2018, Revised 20 November 2018, Accepted 27 November 2018

DOI: $10.6025 /$ jet/2019/10/1/1-9

C2019 DLINE. All rights reserved

\section{Introduction}

In most underdeveloped countries, the vital role of economic and social development is represented by the agricultural domain, so information on crop and plant health is essential for improving the agricultural sector [1]. The farmers are still using traditional methods for agriculture, which results in low yielding of crops and fruits so an improvement solution may be provided by automatic types of machinery. There is a need to implement modern science and technology in agriculture for increasing the yield. The new improved generation of agricultural systems knowledge, models and products aims to achieve a more sustainable and productive agriculture that uses all the knowledge of data acquisition, modeling, computing power and information technology [2-4]. Modernization of agriculture through the use of IoT technology leads to an increase in production by monitoring each of the sensors required for agricultural crops [5]. The monitored parameters are very useful in agriculture and provide information about the state of the crops and the need for irrigation or not [6]. Monitoring environmental factors is a sufficient solution to

Journal of E - Technology Volume 10 Number 1 February $2019 \quad 1$


increase crop yields, as there are other factors that lead to declining productivity such as timely use of chemical fertilizers. The IoT platforms have become an essential part of IoT deployments; therefore there are several types of such platforms and vendors focused on go-to-market strategies.

The IoT characterizes human life, objects, and things. In the present, a lot of devices have joined through the Internet. Nonetheless, by substituting precision agriculture technologies alternatively of traditional agriculture systems can able to get excellent production outcome to maintain the problems $[7,8]$.

In the last years, technology influence agricultural crop production. With the support of sensor that measures a different type of parameters, farmers can see their crops, and if the temperature of the soil is ok [9], soil moisture [10], an occasion of plant diseases and pests in plants [11]. Farmers respond on time to protect crop loss depending on received information from the sensor devices. IoT farmers can have good products based on precision agriculture systems [12].

The main goal of the paper is to illustrate results achieved using a Libelium-based IoT monitoring platform for precision agriculture. Based on the registered parameters, several vital information for monitoring of the precision agriculture, can be available to the farmers.

This article is divided into 5 sections: the first Section contains the introduction, the $2^{\text {nd }}$ Section contains a brief presentation about IoT-based monitoring solutions and the $3^{\text {rd }}$ Section contains information about Libelium precision agriculture platform. The $4^{\text {th }}$ Section illustrates the results of our studies and the last section presents the drawn conclusions.

\section{IoT-based Monitoring Solutions: Related Work}

Climate changes contribute to the issues occurred in agriculture domain therefore the agricultural sector needs to adapt to the new technologies for data monitoring and transmission.

Farmbot is a device used to solve problems in agriculture, but on a small scale [13]. It also has an application that can configure and control Farmbot from your browser, laptop, tablet or mobile phone. The application has real-time manual commands and a module that allows creating routines execution. The application receives the user input commands so that it can create the type of farm. The Message Queuing Telemetry Transport (MQTT) Gateway is the interface between the Web application and the Farmbot device. Decision Support System analyzes the data collected by sensors and based on them they use algorithms that will optimize the events programmed by the system. For example, considering meteorological forecasting, soil moisture and crop type, Farmbot can decide what water needs to be used to irrigate the plants. All information collected is stored in a database [14].

FarmBeats is an IoT platform for agricultural practices that collects data from various sensors and drones with cameras. The FarmBeats system works even when weather conditions are not favorable. Data collected by FarmBeats sensors are stored in a Cloud that also allows them their indefinitely analyze. The architecture of the FarmBeats system consists in different type of sensors necessary for Smart Agriculture and also for the measure of the air quality. Following specific parameters (humidity and $\mathrm{pH}$ ) and collected data are transmitted to the IoT station using a Wi-Fi protocol. The IoT Base Station is powered by solar panels. Un-like other solutions used in agriculture field, the system is equipped with video cam-eras and drones that capture images and send them to the station via a Wi-Fi connection [15].

The British pioneer technology Kevin Ashton proposed in 1999 the term Internet of Things (IoT) defining interconnected devices that can communicate with each other [11]. The Cloud testing platform uses different types of sensors that monitor a lot of parameters used in agriculture and data are transmitted to the producers. The cloud testing environment provides for the platform a This Remote Terminal Units (RTUs) transmit data using GSM (Global System for Mobile Communications)/ or GPRS (General Packet Radio Service) sensors to the Cloud platform, where data is processed in real time and displayed in the Web viewer application with detailed information on power generation and optimized energy revenues. The system can be connected to other systems management for better use of resources, considering certain factors such as energy price, consumption trends and improved risk management [17].

IoT technology is having disruptive impacts on an extensive range of industries including public transport, agriculture, environmental science, and robotics. In many of these fields, IoT is becoming a key enabler of innovation and success and corpora- 
tions are willing to invest in such new technologies.

IoT is applied in several areas such as precision system [18], management of supply chain products [19], smart grid management system, monitoring smart environmental system [20] to minimize consumption of power and enhance performance.

CLAY-MIST measurement index implementation is a state-of-the-art technique used for measurement and monitoring persistence. It consists of a microcontroller board which receives data from the physical layer connected to sensors to monitor agriculture field. It also evaluates the plant growth [21].

Machine-to-Machine (M2M) telemetry software consists of a server architecture that collects data from differently gateways and makes it available for specialized analysis. The server is that part of the software in which all the actual processing data takes place: storing data in the database and give information to the customers during the connection [22].

Libelium-based measurement solutions for precision farming have been used and proven to be effective across the world in numerous case studies on different crops and crops, also taking into account meteorological phenomena so important for Smart Agriculture. [23].

Therefore the increase in productivity and the efficiency of Libelium platforms are also adequate for Romanian agricultural systems and this is the main goal of this paper.

\section{Libelium-based Platform for Precision Agriculture}

In Smart Agriculture, parameters such as soil and air humidity, temperature, pressure, and solar radiation vary from one area to another, and these measurements lead to the need for a very flexible irrigation system to adapt to all weather conditions. In order to have a good irrigation system it is useful to have a communication network sensor to meet any requirements. Wireless sensors are very important for precision farming, being a solution for developing countries [24].

There are many problems related to agriculture, and these problems addressed also in this paper include:

\section{- Irrigation of Crops}

Irrigation is essential in agriculture, especially in areas where precipitation is not sufficient for crops. Nowadays, many irrigation mechanisms, such as dripping and sprinkling by irrigation are the most widely used. The main purpose of these irrigation methods is the controlled distribution of water in the required area if the water could be saved without compromising the water culture requirements. To find out the amount of water needed in agriculture, IoT plays a vital role in providing different solutions [25-28].

\section{- Pesticide used for Productions}

Using fertilizers in an appropriate amount of maximizes crop productivity. Application of fertilizer and distribution of the required amount of fertilizer in the right place can lead to water quality decline and support the growth of algae. The use of IoT technologies is essential support in terms of time management, control of the number of pesticides and fertilizers. [29,30].

\section{- Crop, Soil and Meteorological Parameters}

Monitoring meteorological parameters is an essential stage of agriculture. Knowing the situation of soil, crops and weather phenomena is necessary for farmers because their decisions to irrigate the crop, spray pesticides, apply fertilizers are based on the current state of the vegetables. Sensor network technology is used to measure local climatic parameters as well as to measure soil and plant status [25-27].

The Libelium acquisition platform is composed of modular acquisition nodes (Waspmotes) and the Meshlium device, which acts as IoT-Gateway. The data acquisition modules connect via $4 \mathrm{G} / \mathrm{WiFi}$ to the Internet or another (private) network to which Meshlium is connected and sends data to it. Once parsed in the Meshlium, the IoT gateway stored the data in a MySQL database that ensures local persistence of data. Sending data to Cloud is done through a software component that serializes data to an MQTT broker. Next, through the Adapter component (a software application developed in Python programming language), is basically an MQTT client that subscribes to several topics and stores the data received from the sensor in the database. For data visualization we used Grafana. The data flow architecture is presented in Figure 1. 

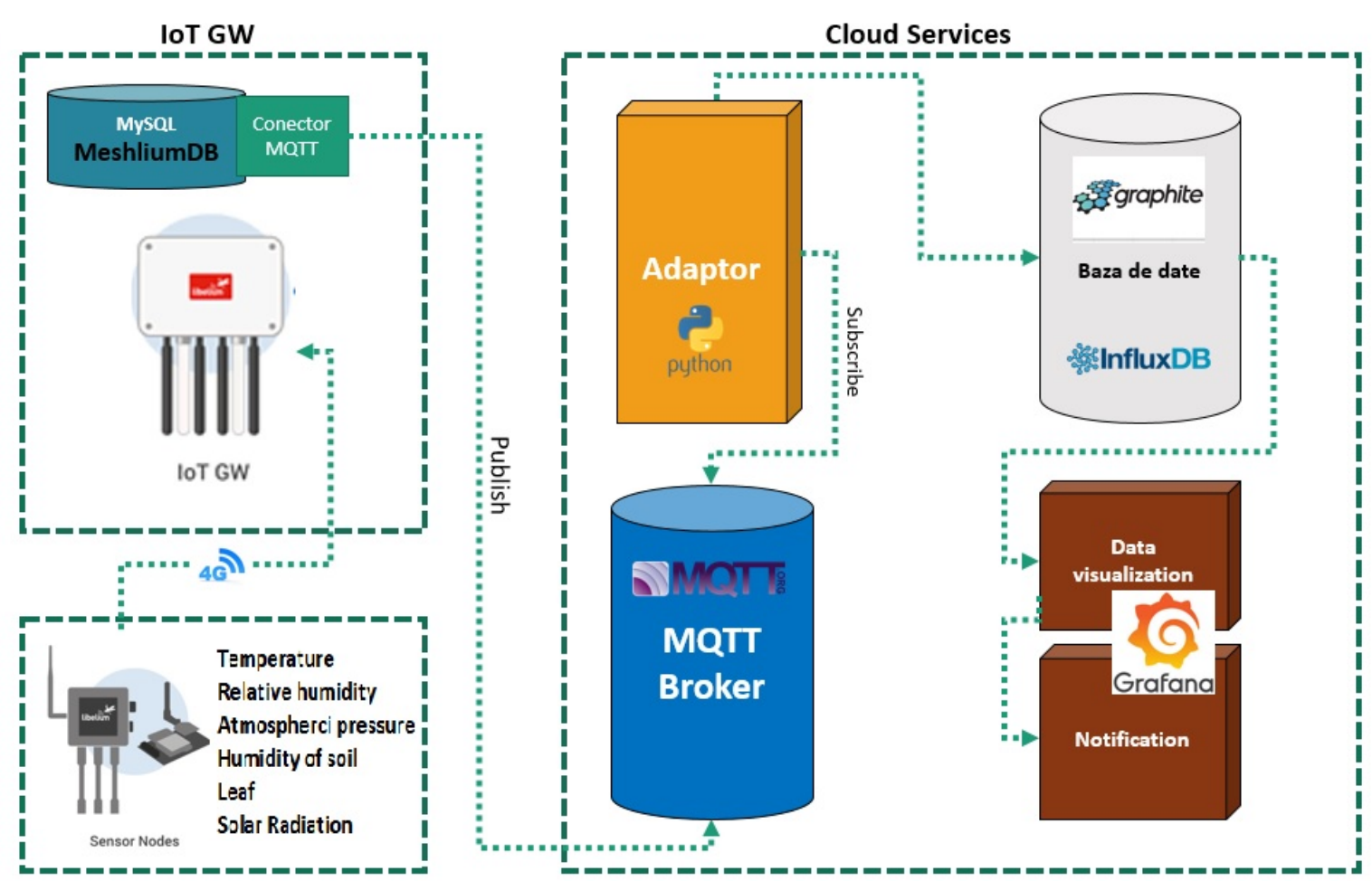

Libelium Smart Agriculture

Figure 1. Data flow architecture

The appropriate settings have been made and the collected data using Libelium platform. Collected data include information regarding air temperature, relative humidity, atmospheric pressure, solar radiation and humidity of soil. The results are presented in Section 4.

\section{Results}

Libelium telemetry is used in this article and we analyze it for a period of one month (March 2018) the parameter for agriculture. This month was taken into consideration since it represents the transition from winter season to spring season. The monitoring of parameters specific to precision farming was carried out in an area cultivated with vineyards in a residential area in Bucharest. As a result of measurements, a series of information on pressure, temperature, solar radiation, humidity is stored, which is stored in a database. In figure 2, 3, 4, 5 and 6 it can be seen the variation of this parameters for one month.

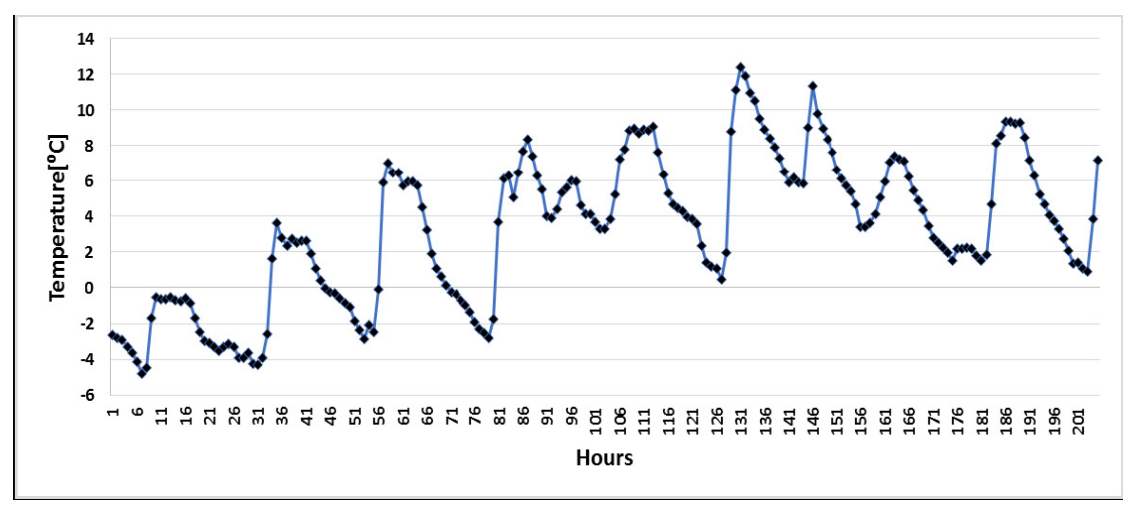

Figure 2. Variation of air temperature in March 2018 
From figure 2 it can be noticed that the transition from winter season to spring season is correctly registered by Libelium monitoring platform. As expected an increase in temperature values is noticeable from the middle of the month which can be an indicator that the farmers can start planting the crops around this time period.

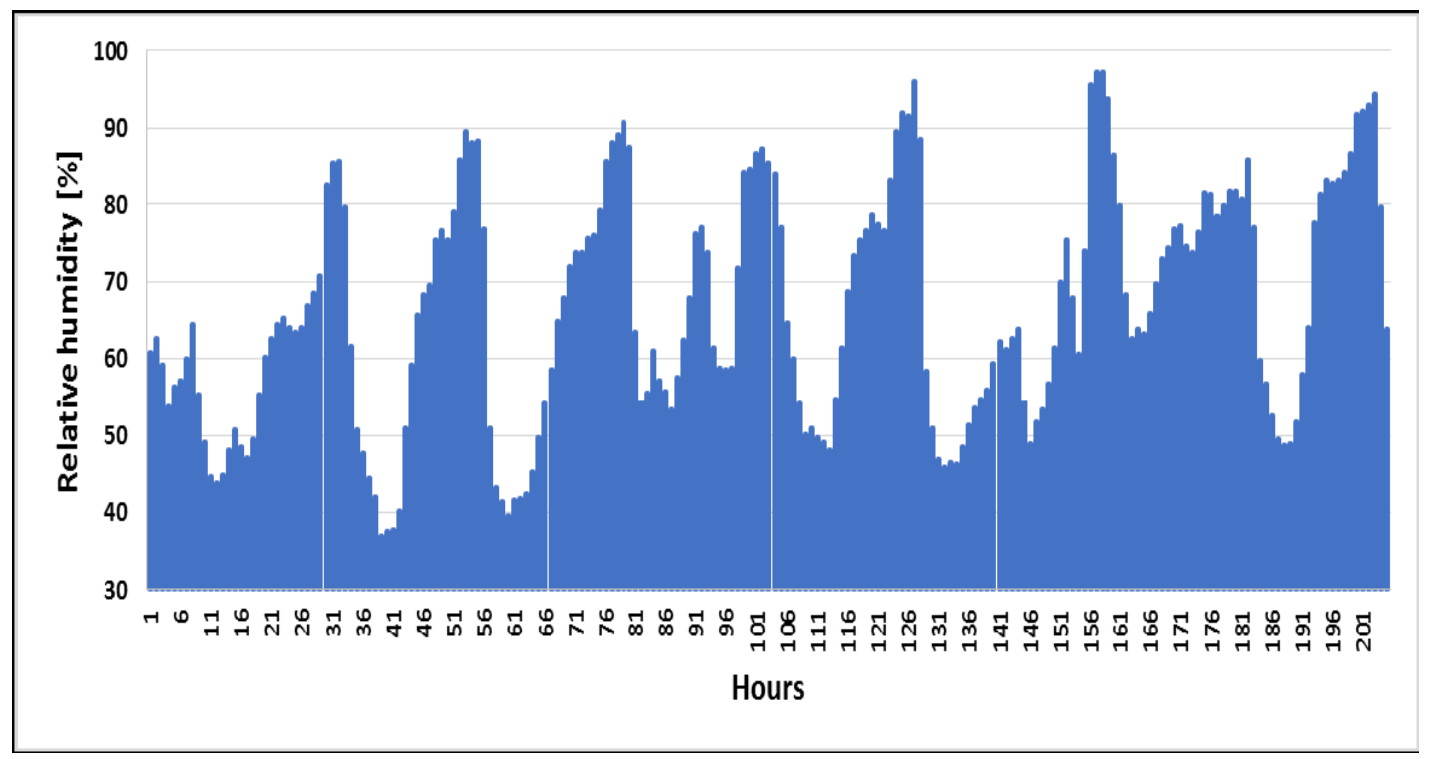

Figure 3. Variation of relative humidity in March 2018

Still, although March is considered a spring month the relative humidity remains approximately constant (figure 3 ) since the temperature values do not vary too much (around $8-12^{\circ} \mathrm{C}$ in Figure 2).

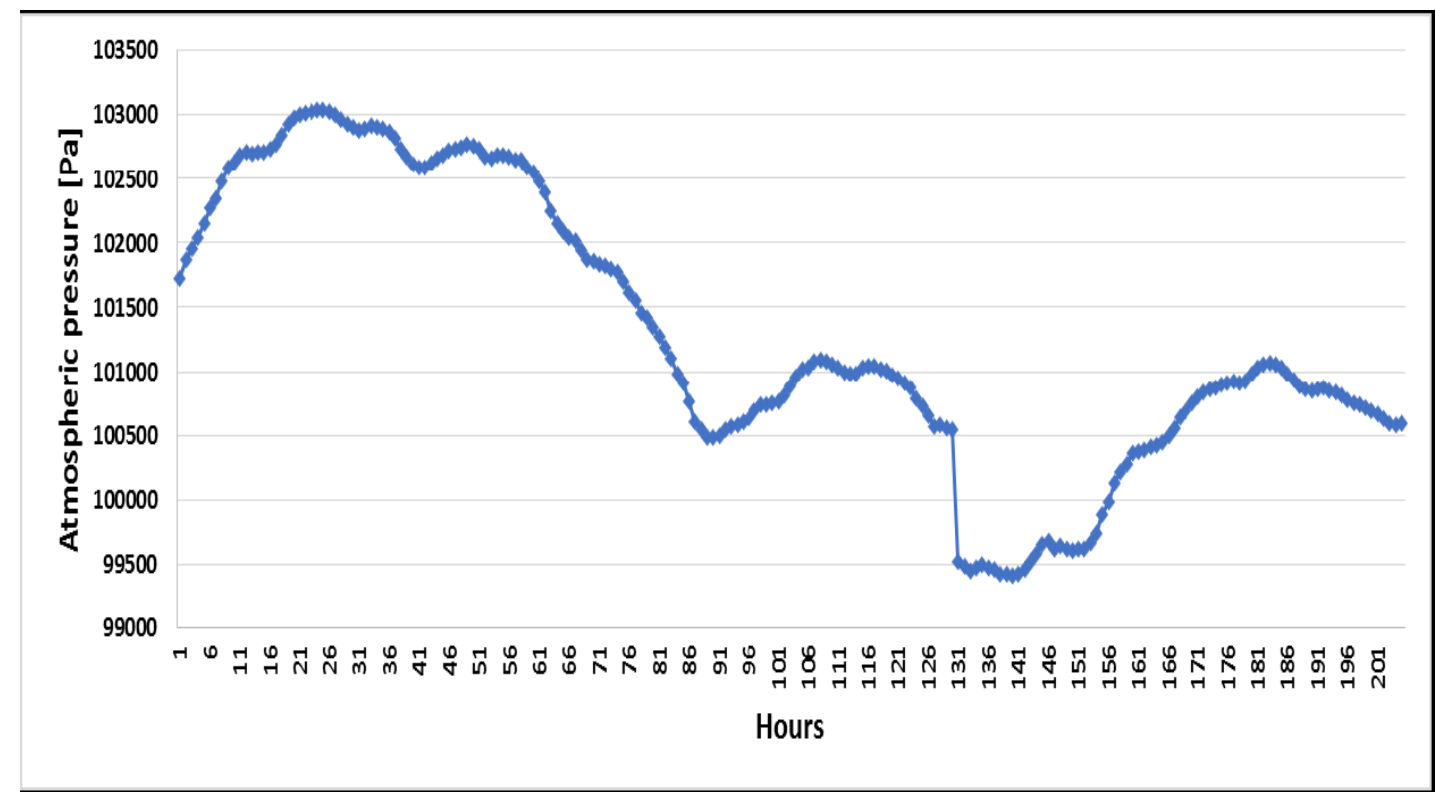

Figure 4 . Variation of atmospheric pressure in March 2018

From the analysis of the graphs (figure 4 and figure 5) we find that the causes of minimum (99500 Pa) and maximum (103000Pa) atmospheric pressures are the daily variation of the air temperature (figure 2) over which overlaps the solar radiation, which produces a phenomenon similar to the tides called the atmospheric tides with a period of 12 hours. 


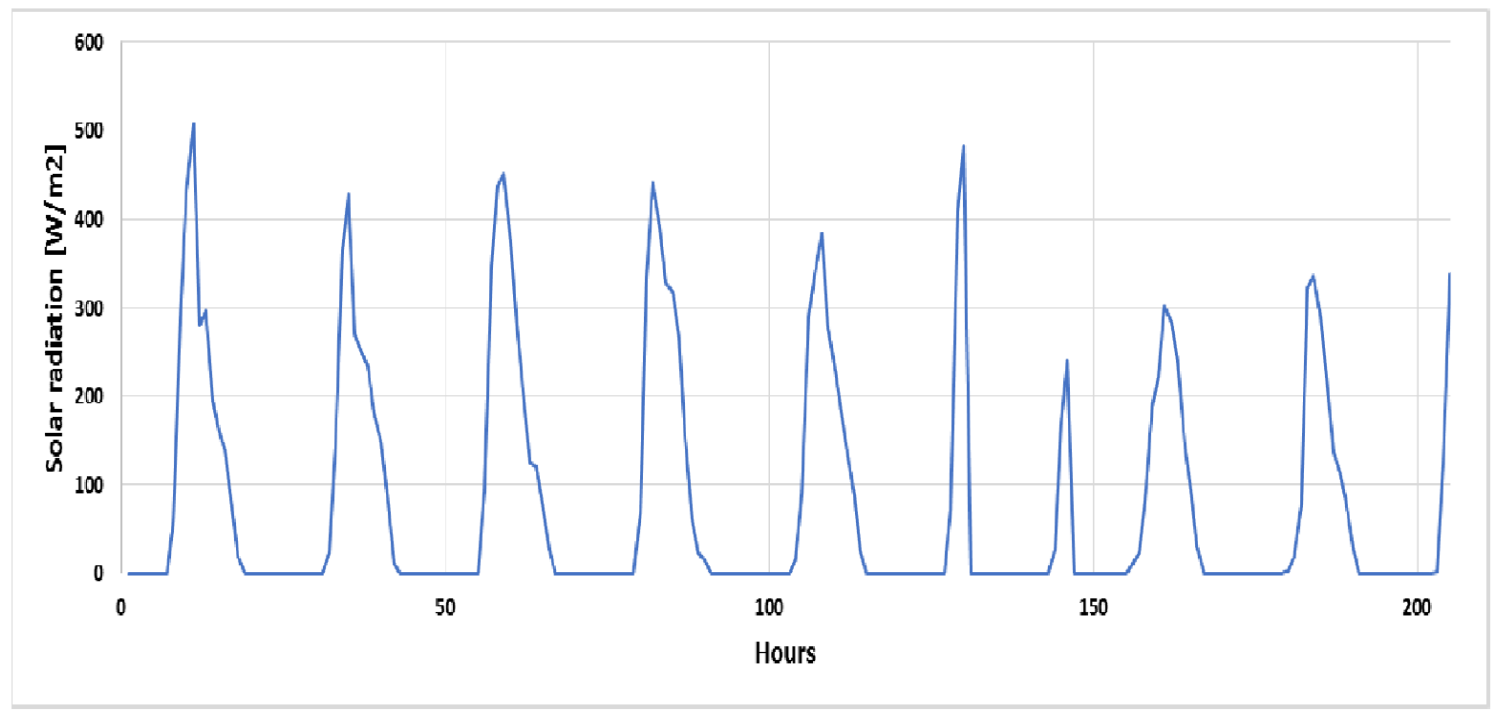

Figure 5. Variation of Solar Radiation in March 2018

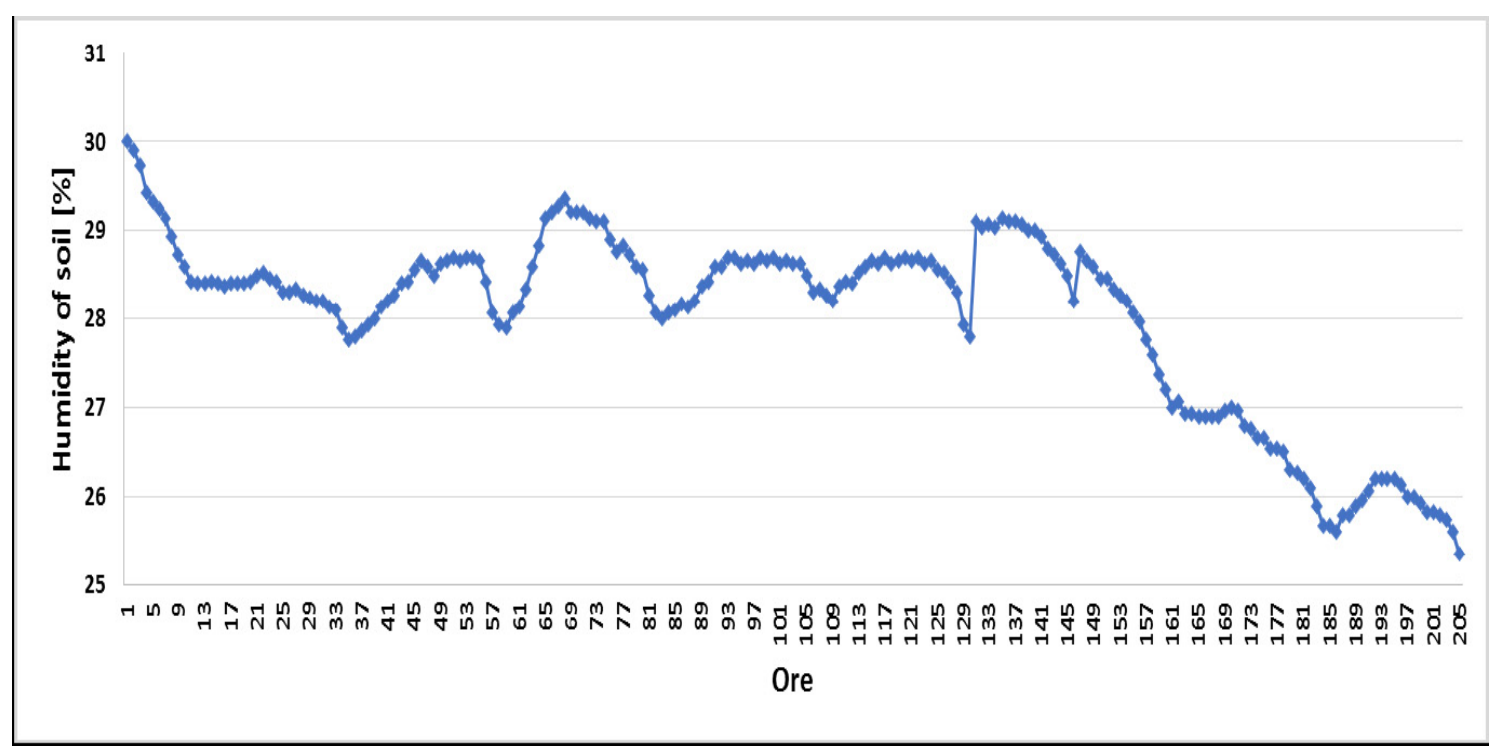

Figure 6. Variation of humidity of soil in March 2018

\begin{tabular}{|l|c|c|c|}
\hline Parameters & Mean Value & Standard deviation & Confidence Level (95.0\%) \\
\hline Air temperature & 4.5 & 0.21 & 0.42 \\
Relative humidity & 74.74 & 0.92 & 1.80 \\
Atmospheric pressure & 101379.2 & 43.13 & 84.80 \\
Solar radiation & 2474.64 & 207.28 & 407.53 \\
Humidity of soil & 31.41 & 0.19 & 0.37 \\
\hline
\end{tabular}

Tabel 1. Statistical analysis of measured parameter 
From the analysis of the graph (figure 6) we find that, according to the technical data from the Libelium Station, the soil moisture is quite low. As conclusion crops irrigation is needed in order to have healthy crops. Table 1 presents a statistical analysis of the parameters measured during this period.

From the analysis of the table no. 1, it is found that for each monitored parameter, confidence level (95\%) is given by the probability that the studied values are within this range.

\section{Conclusion}

Libelium-based platforms used for parameters monitoring in precision agriculture prove their efficiency in various uses cases [24]. This paper aims to illustrate that such platform can be successfully used in Romanian agricultural field and, from the Libelium probes, there can be observed the humidity of soil and other meteorological parameters which are necessary for the growth of plant.

The measured values are transmitted through the Meshlium gateway, and these are validated and then transmitted to the farmers. The emergence of these parameters monitoring and transmission systems, highlights the need for collecting the soil-plant-soil actions that are so useful in agricultural production. Moisture of the monitored soil shows the need for soil irrigation, and the measurement of solar radiation, temperature, relative air humidity give information on crop development in an optimal environment.

For example the transition from winter season to spring season is emphasized by the collected data for air temperature parameter monitored by Libelium platform and farmers can use the values to determine the best period for crop planting. The use of solar panels for powering the Libelium station leads to a very good energy efficiency.

\section{Acknowledgment}

The work presented in this paper has been funded by the Smart Agro project subsidiary contract no. 8592/08.05.2018, from the NETIO project ID: P_40_270, MySmis Code: 105976.

\section{References}

[1] Mao, Y. (2012). Meaning and mode of mobile information services (in Chinese), Information Science, 2, 2012

[2] Mantle, J.,et al. (2017). Towards a new generation of agricultural system data, models and knowledge products: Design and improvement, In: Agricultural Systems, 155, p. 255-268, July 2017.

[3] Jones, J. W., et al. (2017). Towards a new generation of agricultural system models, data, and knowledge products: state of agricultural systems science, Agricultural Systems, 155 p. 269-288, 2017

[4] Janssen, S., et al. (2017). Towards a new generation of agricultural system data, models and knowledge products: Information and communication technology, Agricultural Systems, 155, p. 200-212, 2017.

[5] Zulkifli, C. Z., Noor, N. N. (2017). Wireless Sensor Network and Internet of Things (IoT) Solution in Agriculture, Pertanika Journal of Science and Technology, 25 (1) 91-99, Jan. 2017.

[6] Culman, M, et al (2015). A review of Wireless Sensor Networks for crop field monitoring and considerations for its application in Colombian agriculture, In: Second International Congress of Mechanical Engineering and Agricultural Science (CIIMCA 2015), Colombia, 2015.

[7] What is the Internet of Things (IoT) Homepage https://www.techopedia.com/definition/28247/internet-of-things-iot, accessed March 15 ${ }^{\text {th }}, 2019$;

[8] John Deere Homepage: https://www.deere.com/en/index.html, accesed March 15th, 2019

[9] Lee, I., Lee, K. (2015). Applications, investments, and challenges for enterprises. Business Horizons, 58 (4) 431-440.

[10] Farmbot, Homepage https://farm.bot/, accessed March 16th, 2019 
[11] Joshi, I., et al., (2017). Machine Learning Based Cloud Integrated Farming. In: International Conference on Machine Learning and Soft Computing, p. 1-6, 2017, Vietnam, 2017

[12] Gaitan, N.C., Gaitan, V. G. , Ungurean, I., (2015). A Survey on the Internet of Things Software Arhitecture, International Journal of Advanced Computer Science And Applications, 6,12, : 140-143, Published: DEC 2015, Romania, .

[13] Mekala, M. S., Viswanathan, P., (2017). A novel technology for smart agriculture based on IoT with cloud computing, In: 2017 International Conference On I-SMAC (IOT In Social, Mobile, Analytics And Cloud) (I-SMAC), 75-82, India, 2017.

[14] Alahi, M. E. E., Xie, L., Mukhopadhyay, S., Burkitt, L., (2017). A temperature compensated smart nitrate-sensor for agricultural industry, IEEE Transactions on Industrial Electronics, 64, 9, 7333-7341, SEP 2017,.

[15] Markovic, D., Koprivica, R., Pesovic, U., Ranic, S., (2015). Application of IoT in monitoring and controlling agricultural production, : Acta Agriculturae Serbica 12 (40) 145-153.

[16] Page, K., Dang, Y., Dalal, R., (2013). Impacts of conservation tillage on soil quality, including soil-borne crop diseases, with a focus on semi-arid grain cropping systems, In: Australasian Plant Pathology, 42 (3) 363-377.

[17] Gayatri, M. K., Jayasakthi, J., Mala, G. S. A. (2015). Providing Smart Agricultural Solutions to Farmers for better yielding using IoT, In: 2015 IEEE Technological Innovation in ICT for Agriculture and Rural Development (TIAR), 40-43.

[18] Perles, A., et al. (2018), An energy-efficient internet of things (IoT) architecture for preventive conservation of cultural heritage, FUTURE GENERATION COMPUTER SYSTEMS-THE INTERNATIONAL JOURNAL OF ESCIENCE, 81, 566-581.

[19] Mohanraj, I., Ashokumar, K., Naren, J., (2016). Field Monitoring and Automation using IOT in Agriculture Domain, In: Proceedings of The $6^{\text {th }}$ International Conference On Advances In: Computing and Communications, Book Series: Procedia Computer Science, 93, 931-939, India, 2016.

[20] Ray, P. P., (2016). Internet of Things cloud enabled MISSENARD index measurement for indoor occupants, MEASUREMENT, 92, 157-165.

[21] Mekala, M.S., Viswanathan, P., (2019). CLAY-MIST: IoT-Cloud Enabled CMM index for Smart Agriculture Monitoring System, MEASUREMENT, 134, 236-244 2018, February 2019.

[22] Nakutis, Z., et al. (2019). Remote Agriculture Automation Using Wireless Link and IoT Gateway Infrastructure, In: 26th International Workshop on Database and Expert Systems Applications (DEXA), Electronic 99- 103, Spain, 2015.

[23] Libelium technology, http://libelium.com/downloads/training/webinar_smart_agriculture_libelium_29_06_2017.pdf, accesed March 20th, 2019

[24] http://www.libelium.com/libeliumworld/agriculture/, accesed March 20th, 2019

[25] Holman, I. P., Brown, C., Janes, V., Sandars, D. (2017). Can we be certain about future land use change in Europe? A multiscenario, integrated-assessment analysis, Agricultural Systems, 151, 126-135.

[26] Khatri-Chhetri, A., Aggarwal, P. K., Joshi, P. K., Vyas, S. (2017). Farmers' prioritization of climate-smart agriculture (CSA) technologies, In: Agricultural Systems, 151, 184-191.

[27] Kissoon, D., Deerpaul, H., Mungur, A., (2017). A Smart Irrigation and Monitoring System. In: International Journal of Computer Applications (0975 - 8887), 163 (8), f Mauritius, April 2017.

[28] Nuutinen, M., Schneidman, J. M., Schnetzer, J. (2017). Irrigation in Climate-Smart Agriculture - Challenges and Responses, Webminar, Rome, Italy, June/July 2017.

[29] Walter, A., Fingerb, R., Huberb, R., Buchmanna, N., (2017). Smart farming is key to developing sustainable agriculture, Proceedings of The National Academy of Sciences of The United States of America, 114, 24 6148-6150, DOI: 10.1073/pnas.

$8 \quad$ Journal of E - Technology Volume 10 Number 1 February 2019 


\section{4, WOS:000403179300025}

[30] Pierpaoli, E., Carli, G., Pignatti, E., Canavari, M., (2013). Drivers of Precision Agriculture Technologies Adoption: A Literature Review, In: $6^{\text {th }}$ International Conference on Information and Communication Technologies in Agriculture, Food and Environment (HAICTA2013), Book Series: Procedia Technology, 8, p 61-69, Greece, DOI: 10.1016/j.protcy.2013.11.010, WOS:000335401400010. 